\title{
Optical Möbius Symmetry in Metamaterials
}

\author{
Chih-Wei Chang, ${ }^{1}$ Ming Liu, ${ }^{1}$ Sunghyun Nam, ${ }^{1}$ Shuang Zhang, ${ }^{1}$ Yongmin Liu, ${ }^{1}$ Guy Bartal, ${ }^{1}$ and Xiang Zhang ${ }^{1,2, *}$ \\ ${ }^{1}$ Nanoscale Science and Engineering Center, 3112 Etcheverry Hall, University of California, Berkeley, California 94720-1740, USA \\ ${ }^{2}$ Materials Sciences Division, Lawrence Berkeley National Laboratory, 1 Cyclotron Road, Berkeley, California 94720 , USA \\ (Received 29 April 2010; revised manuscript received 7 October 2010; published 2 December 2010; corrected 6 December 2010)
}

\begin{abstract}
We experimentally observed a new topological symmetry in optical composites, namely, metamaterials. While it is not found yet in nature materials, the electromagnetic Möbius symmetry discovered in metamaterials is equivalent to the structural symmetry of a Möbius strip, with the number of twists controlled by the sign change of the electromagnetic coupling between the meta-atoms. We further demonstrate that metamaterials with different coupling signs exhibit resonance frequencies that depend only on the number but not the locations of the "twists," thus confirming its topological nature. The new topological symmetry found in metamaterials may enable unique functionalities in optical materials.
\end{abstract}

Symmetries play the most fundamental role in science and dictate all physical phenomena, ranging from crystallography, atomic or molecular spectroscopy, chemical reactions, to conservation laws and selection rules, etc. Symmetries can be of different forms; some are apparent and some are hidden. For example, while symmetries of natural molecules or crystals may be easily identified once their structures are known, symmetries beyond apparent spatial geometries are usually obscure. On the other hand, investigating the degeneracy of a system is the most powerful method to unravel the underlying symmetry. For instance, degeneracies of energy levels of an atom in a crystal are determined by the crystalfield symmetry, and the Kramer degeneracy of odd-electrons systems stems from time-reversal symmetry [1].

A three-body system is of special interest for studying the correlation between degeneracy and symmetry because it is a relatively simple system, yet reveals a rich spectrum of phenomena. For example, three particles coupled with three identical mechanical springs exhibit degeneracy that cannot be found in two-coupled oscillators, and three spins with equal antiferromagnetic couplings arranged in a triangular lattice can result in frustrated spin configurations with degenerate ground states [2]. The unique properties of a three-body system make experimental investigations of hidden symmetries possible. Mathematically, the resonance frequency $(\omega)$ of a three-body system (i.e., a trimer) with equal coupling constants (i.e., $\left|\kappa_{12}\right|=\left|\kappa_{23}\right|=$ $\left.\left|\kappa_{13}\right|=\kappa\right)$ can be expressed as

$$
\omega= \begin{cases}\sqrt{1-\kappa}, \sqrt{1-\kappa}, \sqrt{1+2 \kappa} & \text { for } \kappa_{12} \kappa_{23} \kappa_{13}<0 \\ \sqrt{1+\kappa}, \sqrt{1+\kappa}, \sqrt{1-2 \kappa} & \text { for } \kappa_{12} \kappa_{23} \kappa_{13}>0,\end{cases}
$$

where we have normalized the coupling constants with respect to the resonance frequency of the uncoupled system. Notably, this system always possesses twofold degeneracy regardless of the sign of each individual $\kappa_{i j}$ as long as all the coupling constants are of the same magnitude. On the other hand, whether the degenerate mode occurs at the low or the high frequency depends on the sign of the product of $\kappa_{12} \kappa_{23} \kappa_{13}$, as shown in Fig. 1(a).

While positively coupled systems such as mechanical oscillators are common in nature, systems with uniform negative $\kappa_{i j}$ 's or mixed signs of $\kappa_{i j}$ 's have never been found before. Recently, it has been shown that artificially engineered materials can gain more flexibilities than natural atoms or molecules. These so-called metamaterials offer a new perspective for studying new properties beyond natural materials that give rise to various extraordinary optical phenomena such as negative permeability and negative refraction index [3]. These metamaterials are typically made of metallic resonant "meta-atoms." The most

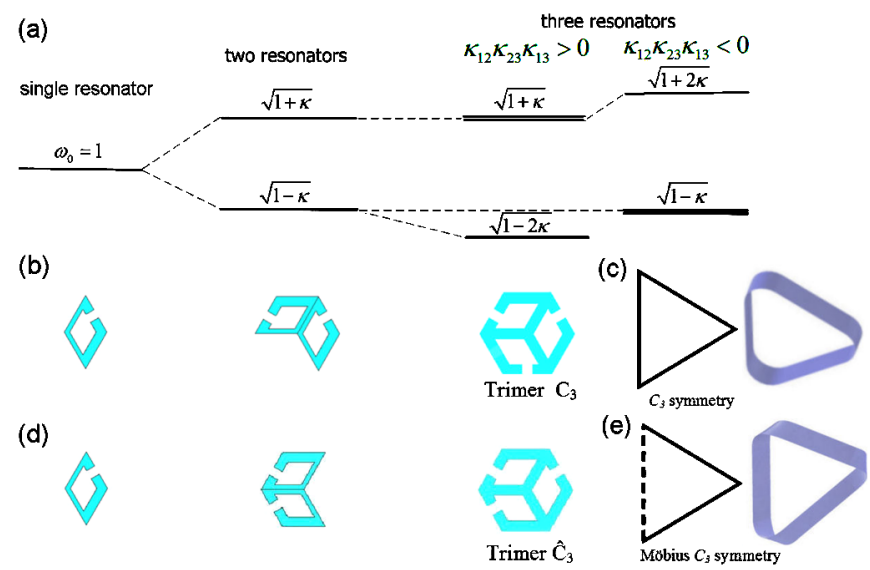

FIG. 1 (color online). (a) Resonance frequency diagram of a single resonator, coupled two-resonator (dimer), and coupled three-resonator (trimer). In general, if there is a degenerate resonance in the trimer, its frequency should overlap with one of the two resonance frequencies of the constituent dimers. (b),(c) A metamolecular system representing a uniform negative coupling configuration in which the trimer can be symbolized as an equilateral triangle, or equivalently, an ordinary strip with $C_{3}$ symmetry. (d),(e) A metamolecular system displaying mixed signs of couplings in which the trimer can be symbolized as a Möbius strip with topological $C_{3}$ symmetry. 
commonly investigated constituent meta-atom is a split-ring resonator, where the resonance frequency is determined by the lumped elements of inductor and capacitor. When two meta-atoms couple to each other (i.e., a dimer), two new hybridized modes will be formed [4]. In particular, the interplay between electric and magnetic dipolar interactions allows tuning of the magnitudes and even the signs of the coupling constants among the constituent meta-atoms [5-7]. In Figs. 1(b) and 1(d), we utilize two kinds of trimers to realize systems with nontrivial coupling configurations through a careful design of the electromagnetic couplings between meta-atoms. We will experimentally demonstrate that these trimers can exhibit $C_{3}$ symmetry and Möbius $C_{3}$ symmetry shown in Figs. 1(c) and 1(e), respectively.

It follows from Eq. (1) and Fig. 1(a) that the degenerate resonance of a trimer will overlap with one of the resonances of the corresponding dimer. In contrast, the nondegenerate resonance in both cases cannot be matched to any resonance in the dimers. Therefore, by comparing the resonance frequencies of a trimer with those of the corresponding dimers, we can identify the degenerate frequency and the sign of the product of $\kappa_{12} \kappa_{23} \kappa_{13}$. Experimentally, the metamolecule arrays were fabricated using standard electron-beam lithography followed by thin metal film depositions and lift-off processes. To identify the resonance frequency of the metamolecules, a Fouriertransform infrared spectrometer was employed for transmission measurements [8]. Because purely electrical interactions cannot lead to negative couplings [5], we focus on the degeneracy and the hidden symmetries of the magnetic resonances of the metamaterial trimers and their constituent dimers. These resonances are manifested as dips in the measured transmission spectra of the metamolecular samples at different incident polarizations.

We have first investigated trimer $\mathrm{C}_{3}$ in Fig. 2. Because trimer $\mathrm{C}_{3}$ exhibits a threefold rotational $\left(C_{3}\right)$ symmetry, a degenerate resonance is expected. We identify the resonance at $94 \mathrm{THz}$ to be the only magnetic resonance for trimer $\mathrm{C}_{3}$, and it does not depend on the incident polarization. According to the conservation of degrees of freedom, a trimer should exhibit three magnetic resonances. But due to $C_{3}$ symmetry, there is an optically dark mode that cannot be excited by a normal incident plane wave. Thus the resonance at $94 \mathrm{THz}$ must be a twofold degenerate mode. The twofold degeneracy is also confirmed by numerical simulations [8]. Furthermore, we have also experimentally investigated dimer 13, which is a subset of trimer $\mathrm{C}_{3}$, so that the sign of $\kappa_{12} \kappa_{23} \kappa_{13}$ can be unraveled. In Fig. 2, one can see that the degenerate resonance of trimer $\mathrm{C}_{3}$ at $94 \mathrm{THz}$ is much closer to the lower resonance of dimer 13 , indicating that $\kappa_{12} \kappa_{23} \kappa_{13}=\kappa_{13}^{3}<0$. Indeed, this is also confirmed by numerical simulations on dimer 13 wherein the frequency of the antisymmetric mode $(87 \mathrm{THz})$ is lower than that of the symmetric mode $(107 \mathrm{THz})$; thus, $\kappa_{13}<0$. Therefore, a system with uniform negative $\kappa_{i j}$ 's is now realized in trimer $\mathrm{C}_{3}$.

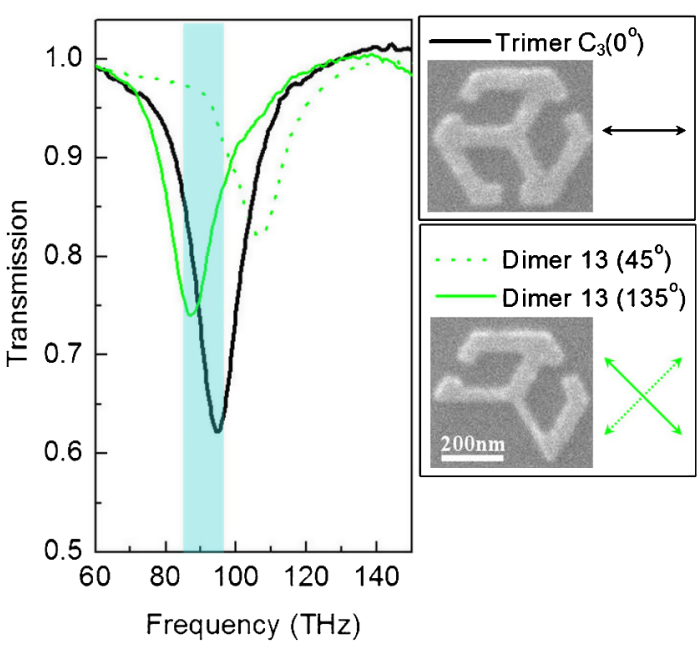

FIG. 2 (color online). The transmission spectra of trimer $\mathrm{C}_{3}$ and its constituent dimer (dimer 13). The number in the parenthesis and the arrows denote the incident polarization angle. The polarizations are chosen only to reveal the resonance frequencies clearly. Trimer $\mathrm{C}_{3}$ spectrum shows a dips at $94 \mathrm{THz}$. Dimer 13 shows an antisymmetric mode at $87 \mathrm{THz}$ and a symmetric mode at $107 \mathrm{THz}$; thus, $\kappa_{13}<0$. The shaded area highlights the region where trimer $\mathrm{C}_{3}$ and dimer 13 exhibit overlapping resonances, indicating that the degenerate resonance of trimer $\mathrm{C}_{3}$ occurs at $94 \mathrm{THz}$.

Next we show that the freedom of engineering the signs of the coupling constants allows metamaterials to exhibit topological symmetries that go beyond those of natural materials. Figure 3(a) depicts the spectra of trimer $\hat{\mathrm{C}}_{3}$, and three pairs of coupled split rings, referred to as dimers 12 , 13 , and 23 , representing the three constituent metamolecules of trimer $\hat{\mathrm{C}}_{3}$. The measured trimer $\hat{\mathrm{C}}_{3}$ spectrum shows two dips at 87 and $106 \mathrm{THz}$, corresponding to two magnetic resonances (the resonance at $132 \mathrm{THz}$ is due to electric dipole resonance). However, the conservation of degrees of freedom imposes three magnetic resonances in a threebody metamolecule, indicating that one of the observed resonances must be degenerate. Based on Eq. (1), we can identify the resonance at $106 \mathrm{THz}$ to be the degenerate states as it overlaps with the measured resonances of dimer 13 and dimer 12 (Fig. 3) and the resonance of a dark mode of dimer 23 , which is obtained numerically since it cannot be excited by the incident wave [8]. The fact that the degeneracy occurs at the higher frequency indicates that the product of the coupling coefficients $\kappa_{12} \kappa_{23} \kappa_{13}$ is positive, as is consistent with the detailed analysis of both the measured transmission spectra of the constituent dimers and the numerical simulations; i.e., $\kappa_{12}>0, \kappa_{23}<0, \kappa_{13}<0$ [8].

The observed degeneracy reveals the underlying symmetry of the metamaterial trimers. As expected, the degeneracy of trimer $\mathrm{C}_{3}$ originates from the structural $C_{3}$ symmetry. To better visualize the symmetry, we map it to an equilateral triangle shown in Fig. 1(c) with solid lines representing the negative coupling constants. On the other hand, the symmetry of trimer $\hat{\mathrm{C}}_{3}$ is less trivial. Although the magnitudes of $\kappa_{i j}$ 's are approximately equal, their 


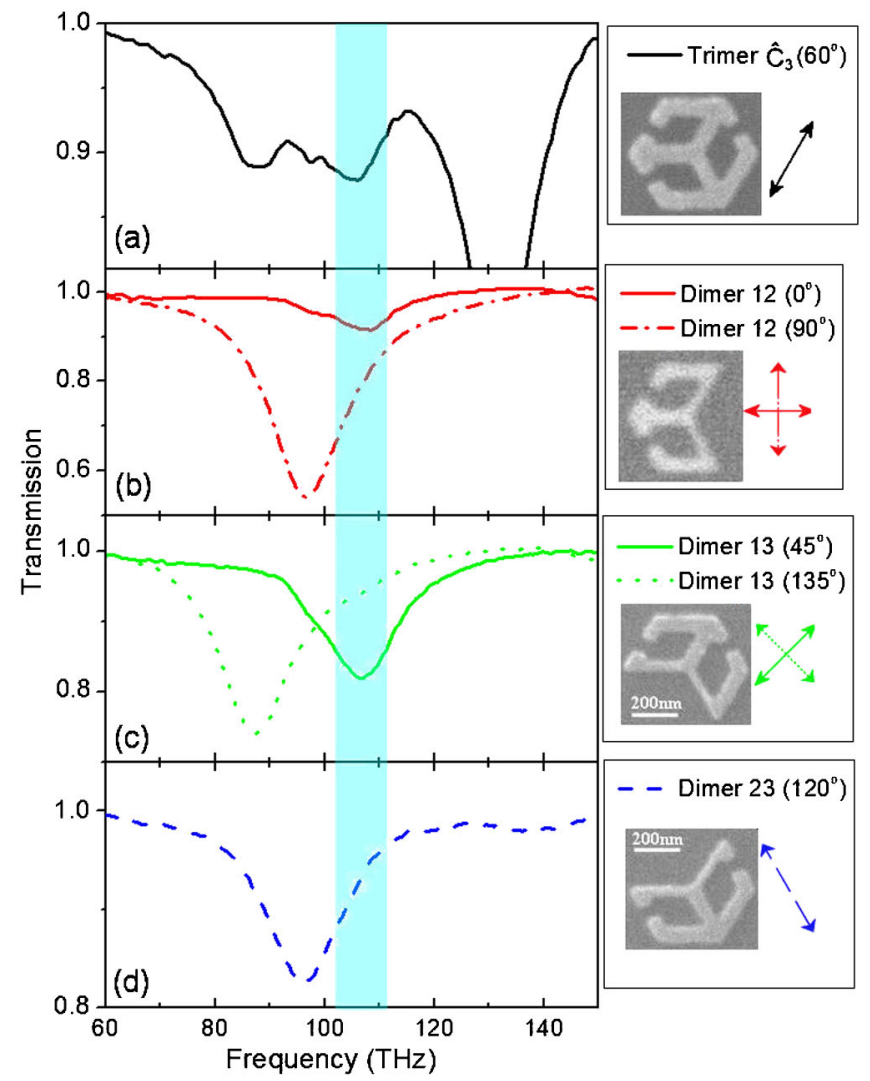

FIG. 3 (color online). The transmission spectra of trimer $\hat{\mathrm{C}}_{3}$ and its constituent dimers. The number in the parentheses and the arrows denote the incident polarization angle. The polarizations are chosen only to reveal the resonance frequencies clearly. (a) Trimer $\hat{\mathrm{C}}_{3}$ spectrum shows two dips at 87 and $106 \mathrm{THz}$, representing two magnetic resonances. (b) Dimer 12 shows a symmetric mode at $96 \mathrm{THz}$ and an antisymmetric mode at $108 \mathrm{THz}$; thus, $\kappa_{12}>0$. (c) Dimer 13 shows an antisymmetric mode at $87 \mathrm{THz}$ and a symmetric mode at $107 \mathrm{THz}$; thus, $\kappa_{13}<0$. (d) Dimer 23 shows an antisymmetric mode at $96 \mathrm{THz}$ and a symmetric mode (not optically active, obtained via simulation) at $106 \mathrm{THz}$; thus, $\kappa_{23}<0$. The shaded area highlights the region where trimer $\hat{\mathrm{C}}_{3}$ and the dimers exhibit overlapping resonances, indicating that the degenerate resonance of trimer $\hat{\mathrm{C}}_{3}$ occurs at $106 \mathrm{THz}$.

signs are mixed; i.e., $\kappa_{23}, \kappa_{13}$ are negative but $\kappa_{12}$ is positive. Thus we can map the symmetry of trimer $\hat{\mathrm{C}}_{3}$ to an equilateral triangle with one dashed line symbolizing the positive coupling, as shown in Fig. 1(e). Since both trimer $\mathrm{C}_{3}$ and trimer $\hat{\mathrm{C}}_{3}$ exhibit twofold degeneracy, the configuration in Fig. 1(e) should exhibit similar $C_{3}$ symmetry as that in Fig. 1(c). However, unlike the apparent $C_{3}$ symmetry in trimer $\mathrm{C}_{3}$, the configuration in Fig. 1(e) does not show apparent geometrical symmetries. We find that this hidden symmetry can be visualized as a topological $C_{3}$ symmetry similar to a Möbius strip in which the rotational symmetry should be accompanied by a sign change of the coupling constant, or equivalently, the front and back sides are in fact the same side in a Möbius strip.

Surprisingly, even though the structural $C_{3}$ symmetry has been destroyed by our intentional design of the asymmetric orientations of the gaps, the degeneracy of trimer $\hat{\mathrm{C}}_{3}$ is still preserved. Thus the symmetries enabled by the metamolecules span a mathematical space much larger than the conventional systems do.

The topological $C_{3}$ symmetry found in trimer $\hat{\mathrm{C}}_{3}$ has no counterparts in any natural materials. Naturally occurring chemical bonds originating from electric interactions cannot exhibit stable positive and negative coupling constants simultaneously. Only spin systems which allow simultaneous ferromagnetic and antiferromagnetic interactions may possess the freedom of displaying opposite signs of coupling constants. However, introducing ferromagnetic interactions to an antiferromagnetic spin system in a triangular lattice (i.e., a frustrated spin system) will destroy the rotational symmetry and remove the degeneracy of the many-body ground state. Here we have shown that the degeneracy of the trimers is preserved even when the coupling constants flip signs. Thus, the topological $C_{3}$ symmetry found in metamolecule trimer $\hat{\mathrm{C}}_{3}$ is a new symmetry beyond those of naturally occurring materials or molecules.

In general, topology possesses a unique feature which only concerns the global property of the system and is insensitive to local differences. In order to elucidate the extraordinary topological effect in metamaterials, we experimentally investigate systems consisting of six-coupled split-ring resonators (i.e., hexamers). Remarkably, analyses of the hexamer systems show that their eigenfrequency exhibits sensitive dependence on the number of positive $\kappa_{i j}$ 's (or equivalently, negative $\kappa_{i j}$ 's) but not on their locations [8]. In fact, the eigenfrequencies of a hexamer with even number of positive $\kappa_{i j}$ 's are similar to the energy levels of benzene molecules (Hückel annulenes), whereas for odd number of positive $\kappa_{i j}$ 's the eigenfrequencies correspond to the energy levels of hypothetical Möbius annulenes [9]. Therefore, the number of positive $\kappa_{i j}$ 's in a hexamer system is equivalent to the number of twists in a Möbius strip. Notably, the topological differences between Hückel and Möbius annulenes results in their stability conditions with opposite characteristics that sensitively depend on the number of twists [9].

Figure 4 shows the schematics of the various coupling constants arrangements in the hexamers, where hexamer 0 exhibits all negative coupling constants, hexamer 1 with one positive coupling, 2A and 2B have two positive couplings at different locations, and hexamer 3 has three positive couplings [8]. Figure 4 also shows the corresponding scanning electron microscopy images of the fabricated metamolecules.

The extraordinary topological effect is best illustrated by the measured transmission spectra of hexamer $2 \mathrm{~A}$ and hexamer 2B shown in Fig. 4. For a $y$-polarized incident light, the resonance at $74 \mathrm{THz}$ is identified to be the magnetic resonance for both hexamers having two positive $\kappa_{i j}$ 's but at different locations. Despite the structural differences between the two systems, Fig. 3 shows that not 

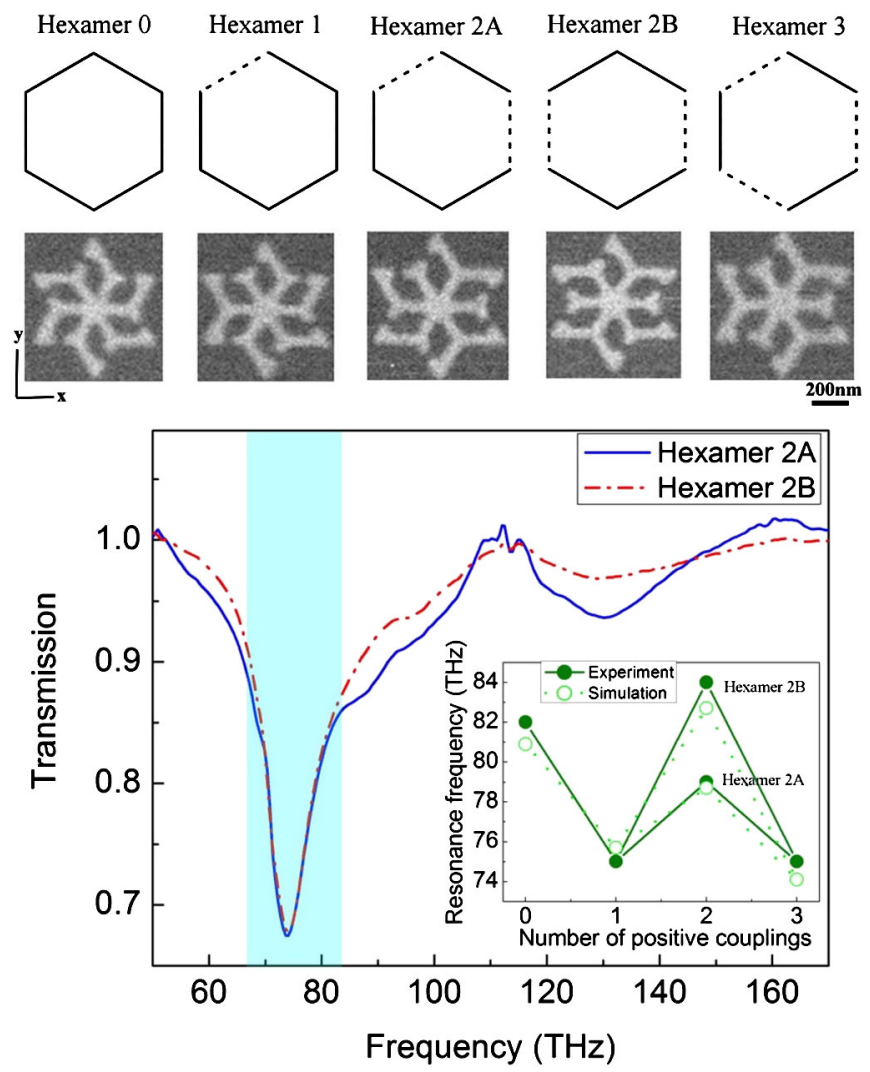

FIG. 4 (color online). Upper row: Schematic graphs which highlight the signs of $\kappa_{i j}$ 's of the hexamers. A solid line denotes a negative $\kappa_{i j}$ and a dashed line denotes a positive $\kappa_{i j}$. Lower row: Corresponding scanning electron microscopy images of the fabricated hexamers. Main panel: Two structurally distinct but topologically identical metamolecules (hexamer 2A and hexamer 2B) show nearly identical transmission spectra (shaded area) for a $y$-polarized incident light, which highlights a topological effect. Inset: The frequencies of the magnetic resonances for an $x$-polarized incident light displaying an oscillation with respect to odd or even number of positive $\kappa_{i j}$ 's in the hexamers, which demonstrates another topological effect (see text). The experimental data and simulation results are shown for comparison.

only the frequency but also the magnitude of the resonances of the two different hexamers overlap significantly at $74 \mathrm{THz}$. The result clearly verifies the topological effect that the resonance of the hexamers does not depend on the location of the twist (i.e., positive $\kappa_{i j}$ ) once its number is fixed. Ideally, the resonance of the hexamers should only depend on whether there are odd or even number of twists and show oscillation behavior, as shown in the inset of Fig. 4. However, due to the effect of the multipolar interactions among the meta-atoms, the resonance frequencies are shifted and the degeneracy among hexamers of the same parity is lifted to some extent. Nevertheless, a trend of oscillation of resonance frequency versus the number of twist is clearly observed in the inset of Fig. 4, indicating the important role the parity of the number of twist plays in the topological hexamers.

Very few natural materials are known to exhibit topological features. Azabenzene molecules such as benzene, pyridine, pyrimidine, pyrazine, and 1,3,5-triazine exhibit point group symmetries similar to those of the hexamers; however, as their coupling constants are all positive, their vibrational spectra do not exhibit similar topological effects as the metamaterials $[10,11]$. We find that the closest analogy to the topological metamaterials may be $\mathrm{NbSe}_{3}$ crystals under special conditions or synthesized Möbius annulenes [12,13]. However, the topological effects in these materials are usually obscured by the strain introduced in the structural twist $[14,15]$. On the other hand, the coupling constants of metamolecules can be arbitrarily varied from positive to negative without any constraints rendering the number of Möbius twists unlimited. More varieties of topological structures that were thus far limited to mathematical imagination can now be realized using metamolecules of different designs. Extending the new symmetries of metamaterials to other kinds of artificial systems such as Josephson junctions will open new avenues for novel phenomena in quantum electronics or quantum optics.

This work was supported by the U.S. Department of Energy under Contract No. DE-AC02-05CH11231 on simulations and fabrication, and by the NSF Nano-scale Science and Engineering Center (NSEC) under Grant No. CMMI-0751621 for optical characterizations.

*Corresponding author: xiang@berkeley.edu

[1] J.J. Sakurai, Modern Quantum Mechanics (AddisonWesley, Reading, MA, 1993).

[2] S. T. Bramwell and M. J.P. Gingras, Science 294, 1495 (2001).

[3] V. M. Shalaev, Nat. Photon. 1, 41 (2007).

[4] E. Prodan, C. Radloff, N. J. Halas, and P. Nordlander, Science 302, 419 (2003).

[5] T. Li et al., Opt. Express 17, 11486 (2009).

[6] H. Liu et al., Phys. Rev. Lett. 97, 243902 (2006).

[7] N. Liu, L. W. Fu, S. Kaiser, H. Schweizer, and H. Giessen, Adv. Mater. 20, 3859 (2008).

[8] See supplementary material at http://link.aps.org/ supplemental/10.1103/PhysRevLett.105.235501 for detailed information.

[9] H. S. Rzepa, Chem. Rev. 105, 3697 (2005).

[10] F. Billes, H. Mikosch, and S. Holly, THEOCHEM 423, 225 (1998).

[11] A. D. Boese and J. M. L. Martin, J. Phys. Chem. A 108, 3085 (2004).

[12] S. Tanda et al., Nature (London) 417, 397 (2002).

[13] D. Ajami, O. Oeckler, A. Simon, and R. Herges, Nature (London) 426, 819 (2003).

[14] C. Castro et al., J. Am. Chem. Soc. 127, 2425 (2005).

[15] T. Matsuura, T. Tsuneta, K. Inagaki, and S. Tanda, Phys. Rev. B 73, 165118 (2006). 